\title{
Foreword, with a Dedication to Andreas Weber
}

\author{
Matthew England • Wolfram Koepf • \\ Timur Sadykov • Werner M. Seiler • \\ Thomas Sturm
}

Published online: 5 June 2020

(C) Springer Nature Switzerland AG 2020

The International Workshop on Computer Algebra in Scientific Computing (CASC) is an annual conference on Computer Algebra Systems, the underlying algorithms for Symbolic Computation, and the role of both within Scientific Computation and its applications. In 2019 the 21st CASC conference was held in Moscow, Russia, hosted by the Plekhanov Russian University of Economics. This special issue of Mathematics in Computer Science contains selected work that was presented at the conference. CASC maintains a web presence at the following URL. http://www.casc-conference.org

\footnotetext{
M. England ( $\varangle)$

Coventry University, Coventry, UK

e-mail: matthew.england@ coventry.ac.uk

W. Koepf · W. M. Seiler

Universität Kassel, Kassel, Germany

e-mail: koepf@mathematik.uni-kassel.de

W. M. Seiler

e-mail: seiler@mathematik.uni-kassel.de

T. Sadykov

Plekhanov Russian University, Moscow, Russia

e-mail: sadykov1976@mail.ru

T. Sturm

CNRS, Inria, and The University of Lorraine, Nancy, France

e-mail: thomas.sturm@loria.fr

T. Sturm

MPI Informatics and Saarland University, Saarbrücken, Germany

e-mail: sturm@mpi-inf.mpg.de
} 


\section{Scope of CASC}

1. The theory of symbolic computation and its implementation in computer algebra systems. This includes, but is not limited to:

- new methods for exact computation;

- symbolic algorithms and their complexity;

- automated reasoning in algebra and geometry;

- parallel symbolic computation;

- symbolic methods for differential, differential-algebraic and difference equations;

- symbolic methods for polynomial equations and inequalities;

- embedding of computer algebra within different software and hardware environments.

2. All other areas of scientific computing in regards to their benefit from or use of computer algebra methods and software. This includes, but is not limited to:

- the use of computer algebra in approximate computation;

- computer algebra methods in big data analytics;

- computer algebra methods for simulation and modelling.

3. Applications of the above throughout the natural sciences and engineering.

\section{Publication at CASC}

CASC has a long history of publishing conference proceedings as volumes of Springer's Lecture Notes in Computer Science (LNCS). The proceedings from CASC 2019 were recently published as volume 11661. However, following requests from participants, CASC 2019 evolved to offer a variety of participation options to choose in advance of the conference:

1. Participation via submission of a shorter work to the LNCS proceedings.

2. Participations via submission of a full journal article to this special issue.

3. Participation via a short abstract to be distributed only at the conference.

Those authors who participated via the third option were then given the opportunity to submit an article to the special issue shortly after the conference finished.

The new publication options were designed to support the broad community of CASC which covers both mathematicians and computer scientists from all over the world. It proved popular with a marked increase in submissions.

This special issue contains ten accepted papers, all of which received at least three reviews and were agreed for acceptance by the editorial team.

Acknowledgements We wish to first thank the host institution of the CASC 2019 conference, the Plekhanov Russian University of Economics, and the CASC 2019 Local Organizing Committee: Vitaly Minashkin, Timur Sadykov, Vitaly Krasikov, Olga Kitova, and Timur Bosenko. We also acknowledge the co-PC Chairs and Editors of the LNCS Proceedings Matthew England, Wolfram Koepf, and Evgenii V. Vorozhtsov; the co-General Chairs François Boulier, Vladimir P. Gerdt and Werner Seiler; the co-Publicity Chairs Andreas Weber and Dominik Michel; and Hassan Errami who maintains the website. Finally, we thank all members of the CASC 2019 Program Committee and the external reviewers who contributed their time and knowledge to select and improve the published papers.

CASC 2019 Program Committee: Moulay Barkatou, François Boulier, Changbo Chen, Jin-San Cheng, Victor F. Edneral, Jaime Gutierrez, Sergey A. Gutnik, Thomas Hahn, Jeremy Johnson, Dominik L. Michels, Michael Monagan, Marc Moreno Maza, Veronika Pillwein, Alexander Prokopenya, Georg Regensburger, Eugenio RoanesLozano, Valery Romanovski, Timur M. Sadykov, Doru Stefanescu, Akira Terui, Elias Tsigaridas, Jan Verschelde, and Stephen M. Watt. 
Additional External Reviewers for the Special Issue: Christopher Brown, Alicia Dickenstein, Xiaojie Dou, Ruyong Feng, Dima Grigoriev, François Lemaire, Anton Leykin, Wei Li, Elizabeth Mansfield, Chenqi Mou, Nida Obatake, Alexey Ovchinnikov, Gleb Pogudin, Alexander Prokopenya, Daniel Robertz, Julien Sebag, and Bican Xia.

\section{Dedication}

Sadly, Andreas Weber passed away on March 15th 2020. Andreas studied mathematics and computer science at the Universities of Tübingen, Germany, and Boulder, Colorado, USA. He then worked as a postdoc at the Institute for Computer Science at Cornell University, New York, USA; the University of Tübingen, Germany; and the Fraunhofer Institute for Computer Graphics Research, Germany. Since 2001, he was a professor at the University of Bonn, Germany.

Scientifically, he was considered an authority in the fields of physics-based modeling and simulation, as well as applications of computer algebra in the natural sciences and particularly biology. He was incredibly well-read, possessed an immense wealth of knowledge across different disciplines, and was highly committed to international and interdisciplinary networking. Due to his great hospitality and openness, his group in Bonn developed into a permanent lively meeting place of scientists from different fields from all over the world, where science could take place at its best.

Andreas made many contributions to CASC, attending almost all conferences in its history. He contributed many papers, hosted CASC in Bonn in 2007, was an invited speaker in 2010, a PC member from 2000-2013 and most recently served as Publicity Chair from 2014-2019. We will always remember his good-natured humor and his boundless compassion for science and for others. We will miss his presence very much.

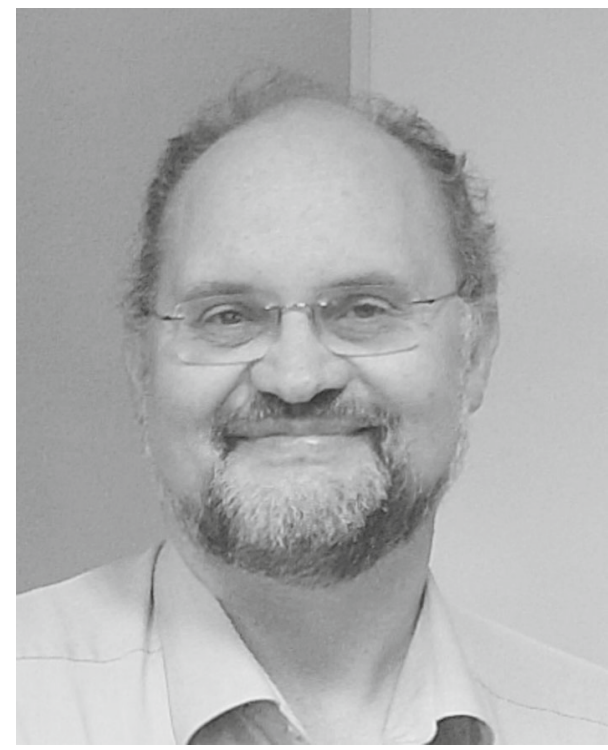

Publisher's Note Springer Nature remains neutral with regard to jurisdictional claims in published maps and institutional affiliations. 